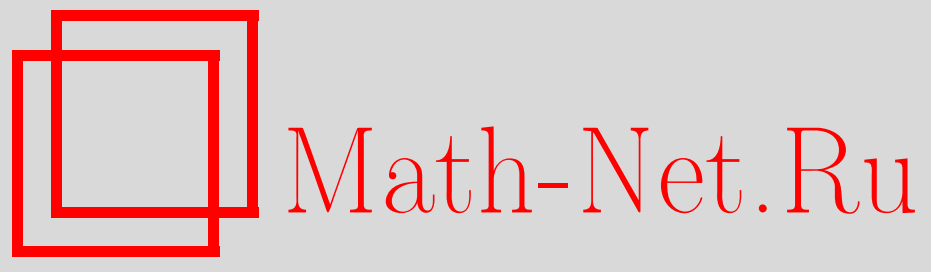

Ф. Калоджеро, Изохронные системы и их квантование, TMФ, 2007, том 152, номер 1, 5-19

DOI: https://doi.org/10.4213/tmf6066

Использование Общероссийского математического портала Math-Net.Ru подразумевает, что вы прочитали и согласны с пользовательским соглашением http://www . mathnet.ru/rus/agreement

Параметры загрузки:

IP: 54.197 .130 .99

26 апреля 2023 г., 15:00:49

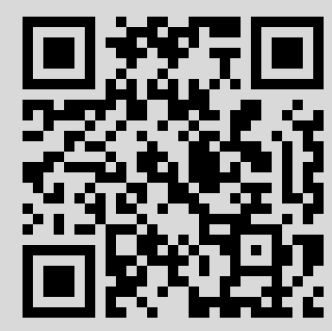




\title{
ИЗОХРОННЫЕ СИСТЕМЫ И ИХ КВАНТОВАНИЕ
}

\begin{abstract}
Дан обзор последних результатов, касающихся классических изохронных систем. Такие системы характеризуются тем, что у них имеется открытая (и, следовательно, полномерная) область их фазового пространства, в которой все решения являются полностью периодическими (т.е. периодическими по всем степеням свободы) с одним и тем же фиксированным периодом (не зависящим от начальных данных, если только они лежат в области изохронности). Представлена техника, позволяющая генерировать такие системы; широкие возможности ее применения подтверждают утверждение о том, что изохронные системы не являются редкими. Описана аналогичная техника, применимая к обширному классу гамильтоновых систем, которая позволяет генерировать изохронные гамильтоновы системы. Также приведены результаты, касающиеся квантованных версий таких систем.
\end{abstract}

Ключевые слова: интегрируемые системы, изохронные системы, квантование.

\section{1. ВВЕДЕНИЕ}

Эта статья основана на докладах, сделанных на IV конференции "Нелинейная физика: теория и эксперимент" (Галлиполи, Лече, Италия, 22 июня - 1 июля 2006 г.) и на Пятом международном семинаре по псевдо-эрмитовым гамильтонианам (Болонья, Италия, 3-5 июля 2006 г.). В статье очерчены базисные идеи, лежащие в основе некоторых недавних находок в области изохронных систем и их квантования, приведены обзоры достижений в этой области и соответствующей литературы.

(Классическая) динамическая система называется изохронной, если у нее имеется открытая, а следовательно, полномерная область в ее естественом фазовом пространстве, в которой все ее решения полностъю периодичны (т.е. периодичны по всем степеням свободы) с одним и тем же фиксированным периодом (не зависящим от начальных данных, если они содержатся внутри области изохронности). В разделе 2 представлен метод, позволяющий сопоставить (автономной) динамической системе (автономную) “ $\omega$-модифицированную” динамическую систему, зависящую от параметра $\omega$ так, что при $\omega=0$ воспроизводится исходная система, в то время как $\omega$ модифицированная система при $\omega>0$ оказывается изохронной (обычно с периодом,

*Dipartimento di Fisica, Università di Roma "La Sapienza", Istituto Nazionale di Fisica Nucleare, Sezione di Roma.

E-mail: francesco.calogero@roma1.infn.it,francesco.calogero@uniroma1.it 
равным $2 \pi / \omega$ или некоторому целому числу $T$ ). Такая техника оказывается применимой к широким классам динамических систем, и это оправдывает утверждение о том, что изохронные системы не редки. Этот факт проиллюстрирован в разделе 3 , где приведен список подобных систем. Там же приведено несколько новых интегрируемых, а на самом деле точно решаемых задач многих тел, заслуживающих названия "золотая рыбка". В разделе 4 описывается еще одна техника, позволяющая преобразовывать гамильтонову систему в $\omega$-модифицированную изохронногамильтонову систему; эта техника находит еще более широкое применение, например, ее можно использовать в произвольной трансляционно-инвариантной (классической) задаче многих тел. Это открывает возможность исследовать квантование многих изохронных систем и найти тем самым, допускают ли (и если да, то до какой степени) эти системы эквидистантный спектр. В разделе 5 содержится новый результат, применимый к цельной, хотя и достаточно специфической, категории таких систем: всякий изохронный гамильтониан, линейный по каноническим импульсам (или по каноническим координатам), порождает после квантования спектр, по крайней мере часть которого оказывается эквидистантной.

\section{2. ТРЮК}

Рассмотрим (автономную) динамическую систему, задаваемую уравнением движения

$$
\frac{d \underline{\zeta}}{d \tau}=\underline{F}(\underline{\zeta}), \quad \underline{\zeta} \equiv \underline{\zeta}(\tau) .
$$

Здесь зависимая переменная $\underline{\zeta} \equiv \underline{\zeta}(\tau)$ может быть вектором, матрицей или тензором, что действительно подтверждает общность выбора такой динамической системы. Тем не менее предполагается, что эта система удовлетворяет следующим двум ограничениям. Во-первых, она должна иметь смысл и в том случае, когда независимая переменная $\tau$ принимает комплексные значения. Поэтому предполагается, что функция $\underline{F}(\underline{\zeta})$, которая задает эту динамическую систему, представляет собой аналитическую (но не обязательно голоморфную) функцию своих аргументов. Исходя из этого, будем считать независимую переменную $\tau$, равно как и зависимые переменные (компоненты $\underline{\zeta}$ ), комплексными числами. Во-вторых, и это менее тривиально, функция $\underline{F}(\underline{\zeta})$, которая, конечно, имеет ту же природу (векторную, матричную или тензорную), что и зависимая переменная $\underline{\zeta}$, обладает масштабным поведением вида

$$
\underline{F}(c \underline{\zeta})=c^{\gamma} \underline{F}(\underline{\zeta}),
$$

где $\gamma$ - рациональное число $(\gamma \neq 1)$. Второе ограничение наложено для простоты и его можно опустить, как будет ясно из рассуждений, приведенных ниже.

Введем замену (независимых и зависимых) переменных, которую далее будем называть "трюк", с помощью следующих формул:

$$
\begin{gathered}
\tau \equiv \tau(t)=\frac{e^{i \omega t}-1}{i \omega}, \\
\underline{z}(t)=e^{i \lambda \omega t} \underline{\zeta}(\tau),
\end{gathered}
$$


где

$$
\lambda=\frac{1}{\gamma-1} .
$$

Здесь и далее новая вещественная независимая переменная $t$ рассматривается как переменная “физического” времени, и точка над символом будет обозначать производную по этой переменной; в дальнейшем рассматривается временна́я эволюция новой зависимой переменной $\underline{z} \equiv \underline{z}(t)$. Предположим далее, что $\omega$ - вещественная (для определенности положительная) константа, с которой связан период

$$
T=\frac{2 \pi}{\omega} .
$$

Из формулы (3а) очевидно следует, что по мере того как $t$ возрастает от своего исходного значения $t=0$, комплексное число $\tau$ (прежняя независимая переменная) движется равномерно в комплексной $\tau$-плоскости круг за кругом против часовой стрелки по окружности $C$, диаметр которой длины $2 / \omega$ лежит на верхней половине мнимой оси, и один конец его находится в начале координат, а другой - в точке $2 i / \omega$; полный оборот совершается за время $T$. Отсюда с необходимостью следует, что $\tau(t)$ - периодическая функция с периодом $T$ :

$$
\tau(t+T)=\tau(t)
$$

Из выражений (3а) и (3б) также следует, что начальные данные для $\underline{z}$ и $\underline{\zeta}$ совпадают,

$$
\underline{z}(0)=\underline{\zeta}(0) .
$$

С помощью (3б) с учетом (3в) легко показать, что новая зависимая переменная $\underline{z}(t)$ теперь эволюционирует в соответствии с новой (автономной) $\omega$-модифицированной динамической системой

$$
\underline{\dot{z}}-i \lambda \omega \underline{z}=\underline{F}(\underline{z}) \text {. }
$$

Эта динамическая система изохронна. В самом деле, обычно имеется открытая область начальных значений $\underline{z}(0)$ такая, что соответствующее решение $\underline{z}(t)$ " $\lambda$-периодично":

$$
\underline{z}(t+T)=e^{2 \pi \lambda i} \underline{z}(t),
$$

и ввиду рациональной природы $\gamma$, а следовательно и $\lambda($ см. $(3 в))$,

$$
\lambda=\frac{p}{q}
$$

где $p$ и $q$ - взаимно простые целые числа $(q>0)$, откуда очевидно следует, что решение $\underline{z}(t)$ периодично по $t$ с периодом, кратным $T$ :

$$
\underline{z}(t+q T)=\underline{z}(t) .
$$

Соотношение (8) явно следует из (3б) и (5), если функция $\zeta(\tau)$ комплексной переменной $\tau$ не имеет точек ветвления внутри (замкнутого) круга $D$ с границей в виде 
окружности $C$ в комплексной плоскости аргумента $\tau$, так что, когда аргумент $\tau$ возвращается к своему значению, обойдя один раз по окружности $C$, эта функция $\underline{\zeta}(\tau)$ также принимает первоначальное значение. Но общее следствие фундаментальной теоремы, гарантирующей существование, единственность и аналитичность решений динамических систем в окрестности их начальных данных, состоит в том, что существует положительное число $\bar{\tau}$ такое, что решение $\underline{\zeta}(\tau)$ системы $(1)$ будет голоморфным при $|\tau| \leqslant \bar{\tau}$. Более того, значение $\bar{\tau}$ зависит от начальных данных, и для автономной системы (1) в общем случае существует открытая область начальных значений $\zeta(0)$ такая, что $\bar{\tau}>2 / \omega$, откуда очевидно следует, что соответствующая функция $\underline{\zeta}(\tau)$ голоморфна в (замкнутом) круге $D$ с границей $C$. Тем самым для соответствующих начальных данных $\underline{z}(0)$ (см. (6)) решение $\underline{z}(t)$ динамической системы (7) периодично, см. (10). Это завершает доказательство изохронности динамической системы (7).

Подчеркнем, что это заключение, справедливое для $\omega$-модифицированной динамической системы (7), полученной из исходной системы (1) с помощью “трюка" (3), не требует от исходной системы (1) никаких специальных свойств, кроме двух, отмеченных выше; в частности, не требуется, чтобы исходная система была интегрируемой. Также из только что приведенных простых соображений следует, что исходная система (1) не обязана быть автономной, даже если мы требуем, чтобы модифицированная система (7) сама была автономной; именно это ограничение и накладывается в дальнейшем, и оно находится в согласии с нашей точкой зрения, утверждающей, что модифицированная система (7) представляет собой “физическую систему”, изохронная эволюция которой как функция вещественного времени $t$ представляет особый интерес. Важно отметить, что исходная система, которая в случае общего положения может иметь неавтономный вид

$$
\frac{d \underline{\zeta}}{d \tau}=\underline{F}(\underline{\zeta} ; \tau), \quad \underline{\zeta} \equiv \underline{\zeta}(\tau),
$$

обладает открытым множеством решений, голоморфных (или, по крайней мере, мероморфных) в круге $D$ комплексной плоскости переменной $\tau$. С помощью этого обобщения можно распространить результаты, изложенные выше, также на случаи, когда функция $\underline{F}$ не удовлетворяет свойству масштабной инвариантности $(2)$ (см. пример 3 в следующем разделе).

С другой стороны, модифицированная система (7) в случае, когда ее начальные данные ограничены на область изохронности (которая, разумеется, сохраняется при временно́й эволюции), оказывается "более, чем суперинтегрируема": свойство суперинтегрируемости обычно предполагает в случае ограниченного движения полную периодичность, но не предполагает, что период фиксирован и не зависит от начальных данных, как это имеет место в случае изохронности. Однако свойство изохронности $\omega$-модифицированной динамической системы (7) обычно выполнено только в ограниченной (пусть даже и в открытой, а потому полномерной) области ее естественного фазового пространства, что следует из приведенных выше соображений. Они также влекут, что комплексификация временно́й эволюции решения 
$\underline{z}(t)$ динамической системы (7) в других областях ее естественного фазового пространства есть прямое следствие комплексификации римановой поверхности (которая связана с зависимостью решения $\underline{\zeta}(\tau)$ задачи (1) от комплексной переменной $\tau)$, поскольку временна́я эволюция $\underline{z}(t)$ в главном отвечает в силу (3) (с точностью до вполне тривиальных периодических множителей) эволюции $\underline{\zeta}(\tau)$, в процессе которой $\tau$ совершает один оборот за другим по окружности $C$ в комплексной плоскости переменной $\tau$, а $\underline{\zeta}(\tau)$ соответственно движется по своей римановой поверхности. Представляет значительный интерес методология [1]-[3] понимания того, как при изменении начальных данных происходит переход от полностью периодичных движений с базисным изохронным периодом к другим, возможно, периодическим движениям со старшими периодами, а также, возможно, к иррегулярным, в некотором смысле, хаотическим движениям. Она задает новую парадигму понимания того, как происходит переход от регулярных к иррегулярным движениям, и, в более общей формулировке, позволяет исследовать иррегулярные (возможно, хаотические) движения. Обсуждение этой проблемы, которая находится в стадии активного развития [4], выходит, однако, за рамки настоящего описания.

Заметим, однако, что если исходная система (1) является интегрируемой, то отсюда обычно следует, что все ее решения оказываются мероморфными функциями независимой переменной $\tau$ или же имеют не более чем конечное число рациональных точек ветвления. Тогда из вышеприведенных аргументов следует, что все решения $\omega$-модифицированной системы (7), а не только те из них, которые лежат в открытой области ее естественного фазового пространства, оказываются полностью периодическими. Исключение, возможно, составляет имеющее более низкую размерность в фазовом пространстве подмножество решений исходной системы (1), для которых сингулярность попадает в точности на окружность $C$. Примечательным примером такой системы, который привел к возникновению понятия "нелинейного гармонического осциллятора" [5], является пример 7 из следующего раздела.

"Трюк” (3) был сначала предложен в специфическом контексте [6], в дальнейшем в силу своей общности он был использован для нахождения и исследования разнообразных $\omega$-модифицированных изохронных систем [7]-[21]. Это оправдывает утверждение о том, что изохронные системы не редки, мы подтвердим это несколькими примерами таких систем в следующем разделе. В заключение этого раздела отметим, что тот же самый подход, позволяющий генерировать $\omega$-модифицированные изохронные динамические системы, может также быть применен (с использованием обобщения указанного "трюка") и в ситуации, более общей, чем указанная выше. Напомним, мы выбрали в качестве отправной точки автономные системы, эволюция которых задается обыкновенным дифференциальным уравнением (ОДУ) или системой ОДУ, и, более того, ограничили рассмотрение системами, в которых масштабные свойства функции $F$ задаются единственным параметром $\gamma$ для всех ее (скалярных) аргументов, см. (2), и, соответственно, множитель перед экспонентой в правой части формулы (3б) содержит единственный параметр $\lambda$, см. (3в). Ни одно из этих ограничений не является необходимым. В цитируемой выше литературе содержится более общее рассмотрение, и мы отсылаем читателя, интересующегося 
аналогичными результатами, которые получены в рамках эволюционных дифференциальных уравнений в частных производных (вместо ОДУ), к работам [22].

\section{3. ИЗОХРОННЫЕ СИСТЕМЫ НЕ РЕДКИ}

В этом разделе дается сжатое описание нескольких примеров изохронных систем с очень краткими комментариями (но с указанием соответствующей литературы).

ПримеР 1. Хорошо известной (см., например, [7]) изохронной системой является одномерная задача нескольких тел, задаваемая гамильтонианом

$$
H(\underline{z}, \underline{p})=\frac{1}{2} \sum_{n=1}^{N}\left(p_{n}^{2}+\omega^{2} z_{n}^{2}\right)+\frac{1}{4} g^{2} \sum_{\substack{n, m=1, n \neq m}}^{N}\left(z_{n}-z_{m}\right)^{-2}
$$

и соответствующими ньютоновскими уравнениями движения

$$
\ddot{z}_{n}+\omega^{2} z_{n}=g^{2} \sum_{\substack{m=1 \\ m \neq n}}^{N}\left(z_{n}-z_{m}\right)^{-3} .
$$

В самом деле, в области вещественных чисел все решения этих уравнений движений оказываются полностью периодическими (изохронными) с периодом $T$, см. (4). Это не вполне верно в области комплексных чисел. В самом деле, рассмотрим (что обычно делается в данной работе) зависимые переменные $z_{n} \equiv z_{n}(t)$ как комплексные, а не вещественные переменные, а также допустим, что "константа связи" $g$ может быть комплексной, всегда тем не менее предполагая вещественность константы $\omega$, которую без ограничения общности можно выбрать положительной. Тогда все движения, совершаемые, разумеется, в комплексной z-плоскости, снова будут полностью периодическими, но период может быть кратным $T$ : хотя в данном случае конфигурация частиц повторяется с периодичностью, равной $T$, индивидуальные частицы могут переставляться в ходе эволюции, так что полный период может оказаться кратным $T$. Этого не может произойти в вещественном случае, когда движение происходит на прямой вещественных чисел, и упорядочение частиц остается неизменным в ходе эволюции из-за сингулярной природы двухчастичного отталкивающего взаимодействия.

Тем самым многочастичная задача, задаваемая таким потенциалом, оказывается изохронной как в вещественной, так и в комплексной области; открытая область начальных данных, в которой наблюдается свойство изохронности, совпадает в этом случае со всем фазовым пространством (кроме более низкоразмерного подмножества начальных данных, приводящих к сингулярным движениям, которые отвечают столкновениям двух и более частиц). И, разумеется, хорошо известно, что эта многочастичная задача суперинтегрируема [7].

ПримеР 2. Рассмотрим более общую многочастичную задачу, задаваемую гамильтонианом

$$
H(\underline{z}, \underline{p})=\frac{1}{2} \sum_{n=1}^{N}\left(p_{n}^{2}+\omega^{2} z_{n}^{2}\right)+\frac{1}{4} \sum_{\substack{n, m=1, n \neq m}}^{N} g_{n m}^{2}\left(z_{n}-z_{m}\right)^{-2},
$$


где $N(N-1) / 2$ “констант связи” $g_{n m}^{2}$ произвольны, а соответствующие ньютоновские уравнения движения имеют вид

$$
\ddot{z}_{n}+\omega^{2} z_{n}=\sum_{\substack{m=1, m \neq n}}^{N} g_{n m}^{2}\left(z_{n}-z_{m}\right)^{-3} .
$$

Эта задача также оказывается изохронной, но только при рассмотрении в комплексной области. В самом деле, недавно было показано, что ньютоновские уравнения движения (13б) задают полностью периодическую эволюцию с периодом $T$, см. (4), если только начальные данные попадают в подходящую (открытую) область, которая, однако, в общем случае включает в себя не только вещественные значения [1], [8].

ПримеР 3. Аналогичная, но значительно более общая изохронная система [9] задается гамильтонианом

$$
\begin{aligned}
H(\underline{z}, \underline{p})= & \frac{1}{2} \sum_{n=1}^{N}\left(p_{n}^{2}+\omega^{2} z_{n}^{2}\right)+\frac{1}{4} \sum_{\substack{n, m=1 \\
n \neq m}}^{N} g_{n m}^{2}\left(z_{n}-z_{m}\right)^{-2}+ \\
& +\frac{1}{4} \sum_{\substack{n, m=1 \\
n \neq m}}^{N} \sum_{k=1}^{K} \frac{1}{(1+k)} f_{n m}^{(k)}\left(z_{n}-z_{m}\right)^{-2(1+k)}
\end{aligned}
$$

и соответствующими ньютоновскими уравнениями движения

$$
\begin{aligned}
\ddot{z}_{n}+\omega^{2} z_{n}= & \sum_{\substack{m=1, m \neq n}}^{N} g_{n m}^{2}\left(z_{n}-z_{m}\right)^{-3}+ \\
& +\sum_{\substack{m=1, m \neq n}}^{N} \sum_{\substack{m=1 \\
n m}}^{K} f_{n m}^{(k)}\left(z_{n}-z_{m}\right)^{-3-2 k} .
\end{aligned}
$$

Здесь $N$ и $K$ - произвольные положительные целые числа, а $(K+1) N(N-1) / 2$ констант связи $g_{n m}^{2}$ и $f_{n m}^{(k)}$ можно положить любыми (даже комплексными) числами.

ПримеР 4. Замечательный общий класс изохронных динамических систем [10] задается ньютоновскими уравнениями движения

$$
\underline{\ddot{z}}+i \omega \underline{\dot{z}}=\sum_{k=1}^{K} f^{(-k)}(\underline{z}, \underline{\dot{z}}+i \omega \underline{z}),
$$

где зависимая переменная $\underline{z} \equiv \underline{z}(t) \equiv\left(z_{1}, z_{2}, \ldots, z_{N}\right)$ представляет собой вектор произвольной размерности $N$, положительное целое число $K$ также произвольно, и $K$ функций $f^{(-k)}(\underline{z}, \underline{z})$ могут быть любыми, за исключением того, что их зависимость от первой (векторной) переменной должна удовлетворять условию масштабирования:

$$
f^{(-k)}(c \underline{z}, \underline{\tilde{z}})=c^{-k} f^{(-k)}(\underline{z}, \underline{\tilde{z}}) \text {. }
$$


ПримеР 5. Изохронная $\omega$-модифицированная версия гравитационной задачи $N$ тел [11] задается ньютоновскими уравнениями движения

$$
\underline{\underline{r}}_{n}+i \omega \underline{\dot{r}}_{n}+2 \omega^{2} \underline{r}_{n}=\sum_{\substack{m=1, m \neq n}}^{N} \frac{M_{m}\left(\underline{r}_{m}-\underline{r}_{n}\right)}{r_{n m}^{3}} .
$$

Обозначения здесь очевидны, особенно если подчеркнуть, что для $\omega=0$ эти уравнения движения совпадают с уравнениями для стандартной гравитационной задачи $N$ тел (если векторы $\underline{r}_{n}$ трехмерны). Свойство изохронности при этом выполняется для любой размерности векторов $\underline{r}_{n}$. Заметим, однако, что при $\omega>0$ векторы $\underline{r}_{n}(t)$ обязаны быть комплексными.

ПримеР 6. Система из $N+M$ осцилляторов, задаваемая уравнениями эволюции

$$
\begin{aligned}
\dot{x}_{n}-i p_{n} x_{n} & =f_{n}(\underline{x}, \underline{y}), & n & =1, \ldots, N, \\
\dot{y}_{m}+i q_{m} y_{m} & =g_{m}(\underline{x}, \underline{y}), & & m=1, \ldots, M,
\end{aligned}
$$

где $p_{n}, q_{m}$ - неотрицательные (или неположительные) целые числа, является изохронной, если $N+M$ функций $f_{n}(\underline{x}, \underline{y}), g_{m}(\underline{x}, \underline{y})$ от $N$-мерного вектора $\underline{x}$ и $M$-мерного вектора $\underline{y}$ голоморфны при $\underline{x}=\underline{0}, \underline{y}=\underline{0}$, представляют собой многочлены (произвольной степени) от компонент $y_{m}$ вектора $\underline{y}$ и удовлетворяют следующим условиям [12]:

$$
\begin{aligned}
& \lim _{\varepsilon \rightarrow 0} \frac{f(\varepsilon \underline{x}, \varepsilon \underline{y})}{\varepsilon}=\underline{0}, \quad \lim _{\varepsilon \rightarrow 0} \frac{\underline{g}(\varepsilon \underline{x}, \varepsilon \underline{y})}{\varepsilon}=\underline{0}, \\
& \left|\lim _{\varepsilon \rightarrow 0} \frac{f_{n}\left(\varepsilon^{p_{\ell}} x_{\ell}, \varepsilon^{-q_{\ell}} y_{\ell}\right)}{\varepsilon^{1+p_{n}}}\right|<\infty, \quad n=1, \ldots, N, \\
& \left|\lim _{\varepsilon \rightarrow 0} \frac{g_{m}\left(\varepsilon^{p_{\ell}} x_{\ell}, \varepsilon^{-q_{\ell}} y_{\ell}\right)}{\varepsilon^{1-q_{m}}}\right|<\infty, \quad m=1, \ldots, M .
\end{aligned}
$$

ПримеР 7. Нелинейные гармонические осцилляторы [5]. Рассмотрим следующие две (различные при $N>1$ ) системы из $N M$ ньютоновских уравнений движения для $N M$ векторов (произвольной размерности) $\underline{z}_{n m}$ :

$$
\begin{aligned}
& \underline{\ddot{z}}_{n m}-3 i \omega \underline{\dot{z}}_{n m}-2 \omega^{2} \underline{z}_{n m}=c \sum_{\nu=1}^{N} \sum_{\mu=1}^{M} \underline{z}_{n \mu}\left(\underline{z}_{\nu \mu} \cdot \underline{z}_{\nu m}\right), \\
& \underline{\ddot{z}}_{n m}-3 i \omega \underline{\dot{z}}_{n m}-2 \omega^{2} \underline{z}_{n m}=c \sum_{\nu=1}^{N} \sum_{\mu=1}^{M} \underline{z}_{\nu \mu}\left(\underline{z}_{\nu \mu} \cdot \underline{z}_{n m}\right) .
\end{aligned}
$$

Их общие решения несингулярны, и все они полностью периодичны с периодом $T$, cм. (4):

$$
\underline{z}_{n m}(t+T)=\underline{z}_{n m}(t)
$$


Заметим, что в этом случае свойство изохронности выполнено во всем фазовом пространстве за исключением подмногообразия начальных данных низшей размерности, которое отвечает сингулярным решениям. Более того, заметим, что обсуждаемые системы еще не являются наиболее общими системами такого типа, полученными в работе [5].

ПримеР 8. Рассмотрим $N$-частичную задачу на плоскости, характеризуемую следующими ньютоновскими уравнениями движения:

$$
\begin{aligned}
\underline{\ddot{r}}_{n}= & \omega \hat{z} \wedge \underline{\dot{\underline{r}}}_{n}+ \\
& +2 \sum_{\substack{m=1, m \neq n}}^{N} r_{n m}^{-2}\left(\alpha_{n m}+\beta_{n m} \hat{k} \wedge\right)\left[\underline{\dot{r}}_{n}\left(\underline{\dot{r}}_{m} \cdot \underline{r}_{n m}\right)+\underline{\dot{r}}_{m}\left(\underline{\dot{r}}_{n} \cdot \underline{r}_{n m}\right)-\underline{r}_{n m}\left(\underline{\dot{r}}_{n} \cdot \underline{\dot{r}}_{m}\right)\right] .
\end{aligned}
$$

Эта задача изохронна [2], [13]. Здесь (в отличие от большинства предыдущих случаев) координаты оказываются вещественными: $N$ двумерных векторов $\underline{r}_{n}$ лежат в горизонтальной плоскости, вложенной в трехмерное пространство: $\underline{r}_{n} \equiv \underline{r}_{n}(t) \equiv$ $\left(x_{n}, y_{n}, 0\right) ; \underline{r}_{n m} \equiv \underline{r}_{n}-\underline{r}_{m} ;$ постоянный вектор единичной длины $\underline{\hat{z}}$ вертикален, $\underline{z} \equiv(0,0,1)$, скалярное и векторное произведения определены обычным образом (в трехмерном пространстве); при этом $N(N-1)$ (разумеется, вещественных) констант связи $\alpha_{n m}, \beta_{n m}$ можно выбрать произвольным образом, они только должны симметрично зависеть от двух своих индексов: $\alpha_{n m}=\alpha_{m n}, \beta_{n m}=\beta_{m n}$.

Заметим, что несмотря на то что в этом примере силы в ньютоновских уравнениях движения зависят от скоростей, эта модель оказывается гамильтоновой [13].

В специальном случае, определяемом набором констант связи вида

$$
\alpha_{n m}=1, \quad \beta_{n m}=0,
$$

эта модель оказывается интегрируемой (на самом деле, точно решаемой) [7], [23]. В самом деле, полагая $z_{n}=x_{n}+i y_{n}$, мы можем ее переписать в виде

$$
\ddot{z}_{n}=i \omega \dot{z}_{n}+2 \sum_{\substack{m=1, m \neq n}}^{N} \frac{\dot{z}_{n} \dot{z}_{m}}{z_{n}-z_{m}}
$$

Решение при этом задается следующей явной/неявной замечательной формулой:

$$
\sum_{n=1}^{N} \frac{\dot{z}_{n}(0)}{z-z_{n}(0)}=\frac{i \omega}{\exp (i \omega t)-1}
$$

или, эквивалентно,

$$
\frac{\exp (i \omega t)-1}{i \omega} \sum_{n=1}^{N} \dot{z}_{n}(0) \prod_{\substack{m=1, m \neq n}}^{N}\left[z-z_{m}(0)\right]=\prod_{n=1}^{N}\left[z-z_{n}(0)\right]
$$

Последнее соотношение есть (зависящее от $t$ ) полиномиальное уравнение степени $N$ относительно переменной $z$, и $N$ его корней задают положение $N$ (комплексных) координат $z_{n}(t)$ в момент времени $t$ [7], [23]. 
Замечательное свойство системы ОДУ (23), заключающееся в том, что эти уравнения инвариантны как относительно трансляций, так и относительно масштабных преобразований, трансформирующих одновременно зависимые переменные $\underline{z}$ и независимую переменную $t$, дало возможность присвоить этой системе почетное имя "золотой рыбки" [14], которое в дальнейшем было распространено также и на прочие аналогичные системы, см. пример 9 ниже.

Система (21) также интегрируема [24] в случае, когда двухчастичное взаимодействие затрагивает только "ближайших соседей", т.е. при следующем выборе констант связи:

$$
\alpha_{n m}=-\frac{1}{2}\left(\delta_{n, m+1}+\delta_{n, m-1}\right), \quad \beta_{n m}=0 .
$$

Изохронные варианты этой модели были исследованы в работе [15].

Пример 9. Приведем здесь без комментариев другие $N$-частичные задачи типа "золотой рыбки" в своих изохронных версиях. Так как исходные системы, из которых с помощью подходящего варианта "трюка" были получены эти $\omega$-модифицированные модели, интегрируемы (на самом деле, точно решаемы), все эти модели изохронны всюду в своем (естественном) фазовом пространстве за исключением подмногообразия более низкой размерности, которое отвечает траекториям, попадающим в сингулярности, вызванные столкновениями двух и более частиц. В каждом случае отмечены работы, в которых интересующийся читатель может найти исчерпывающее обсуждение (включающее в себя демонстрацию интегрируемости, а на самом деле, и точной решаемости этих моделей).

Первой моделью типа “золотой рыбки” является модель, описывающаяся уравнением

$$
\ddot{z}_{n}-(2 \lambda+1) i \omega \dot{z}_{n}-\lambda(\lambda+1) \omega^{2} z_{n}=2 \sum_{\substack{m=1, m \neq n}}^{N} \frac{\left(\dot{z}_{n}-i \lambda \omega z_{n}\right)\left(\dot{z}_{m}-i \lambda \omega z_{m}\right)}{z_{n}-z_{m}},
$$

где константа $\lambda$ вещественна и рациональна, см. (9), но в остальном произвольна (указанная модель, очевидно, становится исходной моделью (23) типа “золотой рыбки" при $\lambda=0$ ); разумеется, (здесь и везде ниже) $\omega$ положительна [7].

В качестве второго примера “золотой рыбки" приведем уравнение

$$
\ddot{z}_{n}-3 i \omega \dot{z}_{n}-2 \omega^{2} z_{n}=2 a\left(\dot{z}_{n}-i \omega z_{n}\right)+2 \sum_{\substack{m=1, m \neq n}}^{N} \frac{\left(\dot{z}_{n}-i \omega z_{n}\right)\left(\dot{z}_{m}-i \omega z_{m}\right)}{z_{n}-z_{m}} .
$$

Здесь (и ниже) $a$ - произвольная константа (возможно, комплексная); при $a=0$ эта модель, очевидно, сводится к предыдущей при $\lambda=1$ [16].

Можно также рассмотреть уравнение [17]

$$
\ddot{z}_{n}-3 i \omega \dot{z}_{n}-2 \omega^{2} z_{n}=2 a\left(\dot{z}_{n}-i \omega z_{n}\right) z_{n}+2 \sum_{\substack{m=1, m \neq n}}^{N} \frac{\left(\dot{z}_{n}-i \omega z_{n}-a z_{n}^{2}\right)\left(\dot{z}_{m}-i \omega z_{m}-a z_{m}^{2}\right)}{z_{n}-z_{m}} .
$$


Еще одним примером является уравнение

$$
\begin{gathered}
\ddot{z}_{n}-(3+k) i \omega \dot{z}_{n}-(2+k) \omega^{2} z_{n}=a\left[2 \dot{z}_{n}-(2+k) i \omega z_{n}\right] z_{n}+ \\
+2 \sum_{\substack{m=1, m \neq n}}^{N} \frac{\left(\dot{z}_{n}-i \omega z_{n}-a z_{n}^{2}\right)\left(\dot{z}_{m}-i \omega z_{m}-a z_{m}^{2}\right)}{z_{n}-z_{m}} .
\end{gathered}
$$

Здесь $k$ - произвольное целое число $(k \neq 2)$; при $k=0$ эта модель, очевидно, сводится к предыдущей [18].

Наконец, отметим еще два примера: уравнение

$$
\begin{aligned}
& \ddot{z}_{n}+i \omega \dot{z}_{n}=\left(\dot{z}_{n}+i \omega z_{n}-a\right) \times \\
& \quad \times\left[k i \omega+\frac{a}{z_{n}}+(1+a) \sum_{m=1}^{N} \frac{\dot{z}_{m}+i \omega z_{m}-a}{z_{m}}+2 \sum_{\substack{m=1, m \neq n}}^{N} \frac{\dot{z}_{m}+i \omega z_{m}-a}{z_{n}-z_{m}}\right],
\end{aligned}
$$

см. работу [19], а также уравнение

$$
\begin{aligned}
\ddot{z}_{n}-3 i \omega \dot{z}_{n}-2 \omega^{2} z_{n}=2 a^{2} z_{n}^{3}+ & \\
& +2 \sum_{\substack{m=1, m \neq n}}^{N} \frac{\left(\dot{z}_{n}-i \omega z_{n}-a z_{n}^{2}\right)\left(\dot{z}_{m}-i \omega z_{m}-a z_{m}^{2}\right)}{z_{n}-z_{m}},
\end{aligned}
$$

см. работу [20].

\section{4. ИЗОХРОННЫЕ ГАМИЛЬТОНОВЫ СИСТЕМЫ НЕ РЕДКИ}

Опишем теперь технику, позволяющую сопоставить (автономному) гамильтониану $\omega$-модифицированный (и также автономный) гамильтониан, который окажется изохронным: он сводится к исходному, когда параметр $\omega$ обращается в нуль, а при $\omega>0$ у него имеется открытая, а потому полномерная область фазового пространства, в которой все его решения полностью периодичны с одним и тем же периодом. Класс гамильтонианов, к которым можно применить эту технику, весьма обширен: он включает в себя, например, гамильтониан, описывающий класическую многочастичную задачу с трансляционно инвариантными, но в остальном совершенно произвольными потенциалами, которая в дальнейшем активно используется в качестве иллюстрации к нашим находкам. В этом состоит содержание нашей недавней работы [25]; ниже приводится краткий обзор основных результатов, а более подробное рассмотрение можно найти в [25].

Новый “трюк”. Пусть $H(\underline{p}, \underline{q})$ - немодифицированный гамильтониан, представляющий собой отправную точку нашего "трюка". Основное требование, которое наложено на этот немодифицированный гамильтониан, состоит в том, что он позволяет идентифицировать “коллективную переменную” $\Theta(\underline{p}, \underline{q})$, явно определенную в терминах канонических импульсов $p_{n}$ и координат $q_{n}$, временна́я эволюция которой 
(задаваемая немодифицированным гамильтонианом) на самом деле просто эквивалентна времени как таковому. Поэтому эта величина характеризуется тем, что ее скобка Пуассона с гамильтонианом $H(\underline{p}, \underline{q})$ равна единице:

$$
[\Theta(\underline{p}, \underline{q}), H(\underline{p}, \underline{q})] \equiv \sum_{n=1}^{N}\left[\frac{\partial \Theta}{\partial q_{n}} \frac{\partial H}{\partial p_{n}}-\frac{\partial \Theta}{\partial p_{n}} \frac{\partial H}{\partial q_{n}}\right]=1 .
$$

Тогда $\omega$-модифицированный гамильтониан

$$
\widetilde{H}(\underline{p}, \underline{q} ; \omega)=[1+i \omega \Theta(\underline{p}, \underline{q})] H(\underline{p}, \underline{q})
$$

изохронен.

Заметим, что этот $\omega$-модифицированный гамильтониан обычно комплексен (мы, как и раньше, считаем, что параметр $\omega$ принимает вещественные значения). Конечно, мы предполагаем, что в основном рассматриваются аналитические функции: в самом деле, как и в случае, обсуждавшемся выше, введение новой независимой переменной, связанной с временем $t$ формулой (3а) и рассматриваемой как комплексная переменная, лежит в основе доказательства [25] свойства изохронности $\omega$-модифицированного гамильтониана.

Так как мы ожидаем, что изохронность представляет собой общее свойство $\omega$ модифицированного гамильтониана (32) [25], то в дальнейшем можно ограничиться рассмотрением $N$-частичной задачи, задаваемой гамильтонианом

$$
H(\underline{p}, \underline{q})=\frac{1}{2} \sum_{n=1}^{N} p_{n}^{2}+V(\underline{q})+C,
$$

где произвольная константа $C$ вводится для удобства обозначений (см. ниже), а потенциал $V(\underline{q}) \equiv V\left(q_{1}, q_{2}, \ldots, q_{N}\right)$ предполагается трансляционно-инвариантным:

$$
V(\underline{q}+a) \equiv V\left(q_{1}+a, q_{2}+a, \ldots, q_{N}+a\right)=V(\underline{q}),
$$

где $a$ - произвольная константа, что дает (при инфинитезимальных $a$ )

$$
\sum_{n=1}^{N} \frac{\partial V(\underline{q})}{\partial q_{n}}=0
$$

Из этого условия следует, что коллективная переменная $\Theta$, удовлетворяющая условию (31), задается формулой

$$
\Theta(\underline{p}, \underline{q})=\frac{N Q}{P} .
$$

Здесь и далее центр масс $Q$ и полный импульс $P$ определены следующим образом:

$$
Q \equiv \frac{1}{N} \sum_{n=1}^{N} q_{n}, \quad P \equiv \sum_{n=1}^{N} p_{n}
$$


Изохронный характер $\omega$-модифицированного гамильтониана $\widetilde{H}(32)$ с (33), (35) и (36), был показан в работе [25]. Подчеркнем, что доказательство этого результата не потребовало наложения никаких дополнительных ограничений на исходный гамильтониан системы $N$ частиц, кроме свойства галилеевой инвариантности, см. (34); в частности, не требуется никаких масштабных свойств потенциала $V(\underline{q})$.

В работе [25] обсуждаются несколько примеров $\omega$-модифицированных многочастичных задач для различных потенциалов $V(\underline{q})$ в (33). Здесь мы приведем результаты для простейшего потенциала

$$
V(\underline{q})=0 .
$$

Тогда решение соответствующей задачи с начальными данными находится в явном виде [25]:

$$
\begin{aligned}
& p_{n}(t)=p_{n}(0)+\frac{P(0)}{N}\left\{\left[\frac{1-\alpha e^{-i \omega t}}{1-\alpha}\right]^{1 / 2}-1\right\}, \\
& q_{n}(t)=q_{n}(0)+\frac{N Q(0)}{P(0)}\left[e^{i \omega t} p_{n}(t)-p_{n}(0)\right]+p_{n}(t) \frac{e^{i \omega t}-1}{i \omega},
\end{aligned}
$$

где константа $\alpha$ выражается через начальные значения коллективных переменных $H, P$ и $Q$ с помощью формулы

$$
\alpha=\frac{2 N H(0)}{2 N H(0)-P^{2}(0)} .
$$

Изохронный характер этого гамильтониана (см. (32) с учетом (35), (33) и (37)) теперь очевиден, см. (38); его фазовое пространство разбивается на две области: первую, для которой $|\alpha|<1$, а решения полностью периодичны с периодом $T$, см. (4), и вторую, для которой $|\alpha|>1$, а решения полностью периодичны с периодом $2 T$, в то время, как на сепаратрисе, разделяющей эти два сектора и задаваемой соотношениями

$$
|\alpha|=1, \quad \alpha=e^{i \vartheta}, \quad \operatorname{Im} \vartheta=0,
$$

уравнения движения оказываются сингулярными при (вещественном) времени $t_{\mathrm{s}}=$ $\vartheta / \omega(\bmod T)$, поскольку коллективная координата $P$ в этот момент времени обращается в нуль, $P\left(t_{\mathrm{s}}\right)=0$, см. (38a), что приводит к расходимости $\Theta(35)$ и $\widetilde{H}(32)$.

Завершая этот раздел, подчеркнем, что, хотя этот пример и является весьма специальным, представленный результат (доказанный в работе [25]) оказывается достаточно общим ввиду отсутствия какого-либо ограничения на потенциал $V(\underline{q})$.

\section{5. КВАНТОВАНИЕ ИЗОХРОННЫХ ГАМИЛЬТОНИАНОВ}

Изохронные гамильтоновы системы обеспечивают большой простор для исследования различных систем квантования. Эти модели уже пригодились для исследования некоторых интригующих аспектов квантования [26]. Естественная догадка состоит в том, что такие системы, по крайней мере, если они изохронны на всем 
естественном фазовом пространстве, должны задавать эквидистантный спектр в каком-нибудь подходящем квантовом описании.

Самые последние находки [2] подтверждают эту догадку, но только для весьма специального класса таких систем. Если гамильтониан $\widetilde{H}(p, q ; \omega)$ изохронен с периодом $T$ и линеен относительно канонических импульсов $p_{n}$ (или, эквивалентно, относительно канонических координат $q_{n}$ ), то квантовый энергетический спектр $E_{k}$ должен быть (по крайней мере, в какой-то своей части) эквидистантен:

$$
E_{k}=\hbar \omega k=\frac{h}{T} k .
$$

Здесь $k$ - произвольное целое число (положительное, отрицательное или равное нулю), а оставшаяся часть обозначений, хочется надеяться, очевидна (см. (4)). Мы говорим, что квантовый спектр эквидистантен, по крайней мере, в какой-то своей части, если собственным значениям энергии $E_{k}$, задаваемым формулой $(41)$, отвечают унивалентные собственные функции оператора, которому при квантовании можно поставить в соответствие изохронный гамильтониан $\widetilde{H}$. В общем случае эти собственные функции имеют в качестве носителя область фазового пространства, в которой решения соответствующих классичсеких уравнений изохронны; такое ограничение на собственные функции возможно ввиду предполагаемой линейности гамильтониана.

Этот результат в значительной степени независим от выбора процедуры квантования. Он имеет место для изохронного гамильтониана даже в том случае, когда в классическом контексте его изохронность выполнена только в открытой, а потому полномерной подобласти естественного фазового пространства; т.е. даже в ситуации, когда поведение его решений оказывается в класическом случае весьма сложным (“хаотическим") в других областях естественного фазового пространства.

\section{Список литературы}

[1] F. Calogero, M. Sommacal, J. Nonlinear Math. Phys., 9 (2002), 483-516.

[2] F. Calogero, J.-P. Françoise, M. Sommacal, J. Nonlinear Math. Phys., 10 (2003), 157-214.

[3] E. Induti, Sul moto nel piano complesso di particelle attratte verso l'origine con forze lineari ed interagenti a due corpi con forze proporzionali ad una potenza intera, negativa, dispari della loro distanza, Tesi di Laurea, Università di Rome "La Sapienza", Dipartimento di Fisica, 2004; F. Calogero, D. Gomez-Ullate, P. M. Santini, M. Sommacal, J. Phys. A, 38 (2005), 8873-8896; nlin.SI/0507024.

[4] P. Grinevich, P.M. Santini, Newtonian dynamics in the plane corresponding to straight and cyclic motions on the hyperelliptic curve $\mu^{2}=\nu^{n}-1, n \in \mathbb{Z}$ : ergodicity, isochrony, periodicity and fractals, nlin.CD/0607031; Y.N. Fedorov, D. Gomez-Ullate, Physica D, 227 (2007), 120-134; nlin.CD/0607028.

[5] F. Calogero, V. I. Inozemtsev, J. Phys. A, 35 (2002), 10365-10375.

[6] F. Calogero, J. Math. Phys., 38 (1997), 5711-5719.

[7] F. Calogero, Classical many-body problems amenable to exact treatments, Lect. Note. Phys. New Ser. m: Monogr., 66, Springer, Berlin, 2001.

[8] F. Calogero, Phys. Lett. A, 293 (2002), 146-150.

[9] F. Calogero, "Partially superintegrable (indeed isochronous) systems are not rare", New Trends in Integrability and Partial Solvability, Proc. NATO Adv. Res. Workshop (Cadiz, 
Spain, 2002), NATO Sci. Ser. II. Math. Phys. Chem., 132, eds. A. B. Shabat, A. GonzalezLopez, M. Manas, L. Martinez Alonso, M. A. Rodriguez, Kluwer, Dordrecht, 2004, 49-77.

[10] F. Calogero, Appl. Anal., 85 (2006), 5-22.

[11] F. Calogero, J. Phys. A, 35 (2002), 3619-3627.

[12] F. Calogero, J.-P. Françoise, "Isochronous motions galore: nonlinearly coupled oscillators with lots of isochronous solutions", Superintegrability in Classical and Quantum Systems, Proc. Workshop (Montréal, Canada, 2003), CRM Proc. Lect. Notes, 37, ed. P. Tempesta et al., Amer. Math. Soc., Providence, RI, 2004, 15-27.

[13] F. Calogero, J.-P. Françoise, Inverse Problems, 17 (2001), 871-878.

[14] F. Calogero, Phys. D, 152-153 (2001), 78-84.

[15] F. Calogero, L. Di Cerbo, R. Droghei, J. Phys. A, 39 (2006), 313-325.

[16] F. Calogero, J. Math. Phys., 45 (2004), 4661-4678.

[17] F. Calogero, S. Iona, J. Math. Phys., 46 (2005), 103515.

[18] M. Bruschi, F. Calogero, J. Math. Phys., 47 (2006), 022703.

[19] M. Bruschi, F. Calogero, J. Math. Phys., 47 (2006), 042901.

[20] F. Calogero, E. Langmann, J. Math. Phys., 47 (2006), 082702.

[21] F. Calogero, J.-P. Françoise, Ann. H. Poincaré, 1 (2000), 173-191; J. Nonlinear Math. Phys., 9 (2002), 99-125; 13 (2006), 231-254; Ф. Калоджеро, Ж.-П. Франсуаз, ТМФ, 137 (2003), 358-374; M. Bruschi, F. Calogero, Phys. Lett. A, 273 (2000), 173-182; 327 (2004), 320-326; F. Calogero, J. Phys. A, 35 (2002), 985-992; 2002, 4249-4256; "Differential equations featuring many periodic solutions", Geometry and Integrability, London Math. Soc. Lect. Notes Ser., 295, eds. L. Mason, Y. Nutku, Cambridge Univ. Press, Cambridge, 2003, 9-20; J. Nonlinear Math. Phys., 11 (2004), 208-222; J. Math. Phys., 45 (2004), 22662279; "Isochronous Systems", Geometry, Integrability and Quantization, Proc. VI Int. Conf. (Varna, Bulgaria, 2004), eds. I. M. Mladenov, A. C. Hirshfeld, Softex, Sofia, 2005, 11-61; "Isochronous systems", Encyclopedia of Mathematical Physics, v. 3, eds. J.-P. Françoise, G. Naber, Tsou Sheung Tsun, Elsevier, Oxford, 2006, 166-172; S. Iona, F. Calogero, J. Phys. A, 35 (2002), 3091-3098; F. Calogero, L. Di Cerbo, R. Droghei, Phys. Lett. A, 355 (2006), 262-270.

[22] М. Мариани, Ф. Калоджеро, ЯФ, 68 (2005), 935-944; Ф. Калоджеро, М. Мариани, ЯФ, 68 (2005), 1710-1717; F. Calogero, A. Degasperis, Stud. Appl. Math., 113 (2004), 91-137; Phys. D, 200 (2005), 242-256.

[23] F. Calogero, Nuovo Cimento B, 43 (1978), 177-241.

[24] M. Bruschi, O. Ragnisco, Inverse Problems, 5 (1989), 983-998; F. Calogero, J. Nonlinear Math. Phys., 11 (2004), 1-6.

[25] F. Calogero, F. Leyvraz, J. Math. Phys., 47 (2006), 042901.

[26] F. Calogero, S. Graffi, Phys. Lett. A, 313 (2003), 356-362; F. Calogero, Phys. Lett. A, 319 (2003), 240-245; J. Nonlinear Math. Phys., 11 (2004), 1-6.

[27] F. Calogero, F. Leyvraz, J. Phys. A, 39 (2006), 11803-11824. 